\title{
INTELLIGENT TOOL CONDITION MONITORING IN HIGH-SPEED TURNING OF TITANIUM Ti-6Al-4V ALLOY
}

\author{
D.A. Fadare ${ }^{1^{*}}$, E.O. Ezugwu ${ }^{2}$ and J. Bonney ${ }^{2}$ \\ ${ }^{1}$ Department of Mechanical Engineering, University of Ibadan, P.M.B. 1, Ibadan, Nigeria. \\ ${ }^{2}$ Machining Research Centre, FESBE, London South Bank University, London SE1 OAA, England.
}

\begin{abstract}
Intelligent Tool Condition Monitoring (TCM) is an essential requirement in the drive towards automated machining operations. In this paper, a Multi-Layered Perceptron (MLP) neural network model has been developed for on-line condition monitoring of tool wear in high-speed turning of Titanium-based alloy (Ti-6Al-4V). Machining trials were conducted for typical rough and finish turning operations with cutting speed $(90-120 \mathrm{~m} / \mathrm{min})$, feed rate $(0.15-0.2 \mathrm{~mm} / \mathrm{rev})$, and depth of cut (0.5 -2.0 mm) using uncoated cemented carbide (K10 grade) inserts with International Standard Organization (ISO) designation "CNMG 120412". The tool maximum flank wear $\left(V B_{\text {max }}\right.$ ), cutting forces (feed force, $F_{x}$, and tangential force, $F_{z}$ ), and spindle motor power were measured during each machining operation. The cutting parameters (cutting speed, feed rate, and depth of cut), and cutting force and spindle power were used in isolation or in combination as input dataset in training the neural network to predict wear land on cutting tool at different stages of wear propagation (light, medium and heavy). The neural network model was designed using Matlab ${ }^{\circledR}$ neural toolbox. Accuracy of model for the prediction of tool wear at different wear stages were evaluated based on the Percentage Error (PE) for both roughing and finishing operations. Results showed that, the heavy wear stage $(P E= \pm 5 \%)$ was predicted more accurately compared to the light $(P E=+5$ to $-10 \%)$ and medium $(P E=+25$ to $-30 \%)$ wear stages. The combination of the force, power signals and cutting parameters improved performance of the model.
\end{abstract}

Keywords: Artificial neutral network, Turning, Ti-6Al-4V alloy; Tool wear, Condition monitoring

\section{INTRODUCTION}

Modern manufacturing technologies are continually tending towards automation of the production process due to increasing demand for high productivity. For automation in metal cutting operations, the Tool Condition Monitoring (TCM) system is an essential requirement for optimisation of machining operations and reduction of cost of tooling and ultimately a reduction in the overall cost of production (Ezugwu et al., 2004). Implementation of TCM systems involve acquisition of different direct process parameters such as cutting forces, vibration, acoustic emission, power, temperature, 
roughness and roundness of the machined surface, which are measured during machining operations. These measured parameters, which are also influenced by the cutting parameters and other external factors are then used to model and predict the tool condition such as tool life, tool wear, wear rate, wear stage and failure mode. The relationship between the measured process parameters and tool wear parameters are known to be complex and nonlinear time-variant (Silva et al., 2000; Silva et al., 1998) and various modelling techniques have been developed.

The earlier work on TCM systems were focused on development of analytical models (Dimla et al., 1997; Lin et al., 2003). The analytical method is complex and hence time consuming. The need for a more accurate and reliable model for TCM has led researchers into exploring other empirical methods such as multiple regression analysis (Ehmann et al., 1997), wavelet analysis (Wang et al., 2004), time series analysis and frequency domain analysis (Bernhard, 2002). These empirical methods have been applied in practical TCM in metalcutting processes with limited degrees of success. These methods are dependent upon large volume of experimental data, costly and time demanding.

In recent years, application of ANN model for condition monitoring of mechanical systems is becoming increasingly popular in many fields of engineering. Such as in fault detection in rotating machinery (Akangah and Wang, 2006), prediction of wear behaviour of $\mathrm{A} 356 / \mathrm{SiC}$ metal matrix composites (Rashed and Mahmoud, 2009), modelling and prediction of cutting tool wear (Deiab et al., 2009), detection of degraded behaviours in wire electrical discharge machining (Portillo et al., 2009, and vibration detection in milling operation (Kuljanic et al., 2009). In metal cutting operations, different process parameters such as cutting forces, acoustic emission, power consumption, temperature and surface roughness have been correlated to tool wear using a wide range of ANN models with different success rates
(Sick 2002; Liu et al., 1998; Dimla et al., 1997; Dimla and Lister, 2000; Ezugwu et al., 1995). Wilkinson et al. (1999) investigated possible inter-relationship between surface roughness and acoustic emission at different tool wear stages for finish face milling of En24 steel.

This paper investigated the feasibility of using cutting force parameters and spindle motor power consumption in isolation and in combination with cutting parameters for development of a neural network-based on-line tool condition monitoring model for high-speed turning of Ti-6Al-4V alloy.

\section{ARTIFICIAL NEURAL NETWORK}

Artificial Neural network (ANN) is a branch of Artificial Intelligence (AI), which belongs to the group of computational algorithms called connectionist model (Azimian, 2005). ANN models are inspired by the biological neural system, with capability to learn, store and recall information based on a given training dataset. They are 'black-box' modeling technique capable of performing non-linear mapping of a multidimensional input space onto another multidimensional output space without the knowledge of the dynamics of the relationship between the input and output spaces. ANN models have been successfully employed in solving complex problems in various fields of application including pattern recognition, identification, classification, speech, vision, and control systems. In recent times, application of ANN model is becoming increasingly popular in modelling of complex engineering problems.

Basically, ANN models consist of multiple connected Processing Elements (PE), which are called nodes or neurons. They consist generally of five basic components: (1) input, (2) weight and biases, (3) summing junction, (4) transfer function, and (5) output. The neurons are arranged in three multiple layers known as input, hidden and output layer. In neural networks, knowledge is acquired during the training or learning process by updating or adjusting the weights in the network through different algorithms. The network weights are upgraded liter- 
arily until the network reproduces desired output or target from a given set of input. The network is trained with either supervised learning (when both input and the desired targets are presented to the network) or unsupervised learning (when the expected targets are not used in the training). The back-propagation algorithm is a supervised training rule with multiple-layer networks, in which the network weights are moved along the negative of the gradient of the Mean Squared Error (MSE) so as to minimize the difference between the network's output and desired target. There are generally four steps in the training process: (1) assembling the training data, (2) designing the network object, (3) training the network, and (4) simulating the network response with new input data sets. After a sufficient training session, which may require considerable computational resources such as memory and time of the computer, the trained network has adequate capabilities to perform non-linear pattern association between input and output variables and can easily predict the output when a new input data set that is not used in the training is presented to the network. ANN models are known to be efficient and less time-consuming in modelling of complex systems compared to other mathematical models such as regression (Lin et al., 2003). Basic theories and applications of ANN can be found in generic texts such as in Picton (2000).

\section{EXPERIMENTAL PROCEDURES}

The machining operations were conducted on an $11 \mathrm{~kW}$ Computer Numerical Controlled (CNC) turning lathe with a speed range from 18 - $1800 \mathrm{rpm}$, which provides a torque of 1411 Nm. A titanium-based, commercially available alpha-beta Ti-6Al-4V alloy was used as the workpiece. The chemical composition and physical properties of the workpiece are given in Tables 1 and 2 respectively. Indexable rhomboid shaped uncoated carbide (K10 grade) inserts with ISO designation CNMG 120412, with nominal chemical composition (by weight): $93.8 \% \mathrm{WC}, 0.2 \%(\mathrm{Ta}, \mathrm{Nb}) \mathrm{C}$ and $6 \%$ Co were used as cutting tool. The mechanical and other properties of the inserts are: Vickers HV Hardness 1760, density 14.95 g.cm ${ }^{-3}$ and substrate grain size $1.0 \mathrm{~mm}$. Tool holder with ISO designation MSLNR 252512 was used to hold the insert and the followings cutting geometry are employed during the trials: approach angle $40^{\circ}$, side rake angle $0^{\circ}$, clearance angle $6^{0}$ and back rake angle $-5^{0}$.

Prior to the machining trials about $6 \mathrm{~mm}$ thickness of the top surface of the workpiece was removed in order to eliminate any skin defect that can adversely affect machining result. The cutting conditions typical of rough and finish turning of titanium-based alloys in manufacturing industry were employed in this investigation (Table 3).

Table 1. Chemical composition (\% wt) of Ti-6Al-4V alloy

\begin{tabular}{llllllllll}
\hline Chemical element & Al & V & Fe & O & C & H & N & Y & Ti \\
\hline Min. & 5.50 & 3.50 & 0.30 & 0.14 & 0.08 & 0.01 & 0.03 & $50 \mathrm{ppm}$ & Balance \\
Max. & 6.75 & 4.50 & - & 0.23 & - & - & - & - & - \\
\hline
\end{tabular}

Table 2: Physical properties of Ti-6Al-4V alloy

\begin{tabular}{|c|c|c|c|c|c|c|}
\hline $\begin{array}{c}\text { Tensile strength } \\
\text { (MPa) }\end{array}$ & $\begin{array}{c}0.2 \% \text { Proof } \\
\text { stress } \\
\text { (MPa) } \\
\end{array}$ & $\begin{array}{l}\text { Elongation } \\
(\%)\end{array}$ & $\begin{array}{l}\text { Density } \\
\left({\left.\mathrm{g} . \mathrm{cm}^{-3}\right)}^{-3}\right.\end{array}$ & $\begin{array}{c}\text { Melting } \\
\text { point }\left({ }^{\circ} \mathrm{C}\right)\end{array}$ & $\begin{array}{c}\text { Thermal } \\
\text { conductivity } \\
\left(\mathbf{W} \cdot \mathbf{m}^{-1} \cdot \mathbf{K}^{-1}\right)\end{array}$ & $\begin{array}{c}\text { Measured } \\
\text { Hardness } \\
\left(\mathrm{HV}_{100}\right) \\
\end{array}$ \\
\hline $900-1160$ & 830 & 8 & 4.50 & 1650 & 6.6 & $\begin{array}{l}\text { Min. }=341, \\
\text { Max. }=363\end{array}$ \\
\hline
\end{tabular}

*Confidence interval (CI) of 99\%, represented by the minimum (Min) and maximum (Max) values. 
Table 3: Cutting conditions

\begin{tabular}{lll}
\hline & & \multicolumn{2}{c}{ Machining Parameters } \\
\hline \multirow{2}{*}{ Roughing: } & Cutting speed $(\mathrm{m} / \mathrm{min})$ & $80,90,100,110$ and 120 \\
& Feed rate $(\mathrm{mm} / \mathrm{rev})$ & 0.2 \\
& Depth of cut $(\mathrm{mm})$ & 2.0 \\
& Cutting speed $(\mathrm{m} / \mathrm{min})$ & $100,110,120$ and 130 \\
Finishing: & Feed rate $(\mathrm{mm} / \mathrm{rev})$ & 0.15 \\
& Depth of cut $(\mathrm{mm})$ & 0.5 \\
& Type & Emulsion oil (Tri-(2-Hydroxyethyl)-Hexahydrotriazine) \\
Cutting & Concentration (\%) & 6 \\
fluid: & Method of delivery & Overhead cooling at an average flow rate of 2.7 L.min ${ }^{-1}$ \\
&
\end{tabular}

The component forces (targential force, $F_{z}$ and feed force, $F_{x}$ ) were measured using a Kistler piezoelectric tri-axial dynamometer (Type 9257B) attached to the tool post. Signals from the dynamometer were passed through charge amplifiers (Type 5001) with in-built low-pass filters of $680 \mathrm{~Hz}$ cut-off frequency. National Instruments data acquisition card (Model NI PCI/PXI-5112) with threshold frequency of 100 $\mathrm{MHz}$ and 2 acquisition channels with one trigger and a desktop PC with Labview ${ }^{\circledR}$ signal processing software were used for automatic logging and processing of the cutting force signals. The signals were captured at sample rate of $100 \mathrm{MHz}$ and a total of 10,000 data points were recorded for each machining operation.

The spindle motor power required during each of the cutting operation was measured using a machine inbuilt multifunction 3-phase power meter. The experimental setup is shown in Figure 1 , while the schematic of the setup is shown in Figure 2.

The maximum width of flank wear land, $\mathrm{VB}_{\max }$, for each machining trials were measured with a Mitutoyo toolmaker microscopy at interval of two minutes during the machining operations until tool life is reached using the International Standard tool rejection criteria for maximum flank wear land $>0.70$ and $>0.40 \mathrm{~mm}$ for roughing and finishing operations respectively. Based on the measured tool wear, the tool is classified into three wear states: light wear, medium wear and heavy wear as shown in Table 4 .

\section{DESIGN OF THE NEURAL NETWORK Structure of the ANN}

The artificial neural networks used in this study are standard multi-layer perceptron designed with MATLAB ${ }^{\circledR}$ Neural Network Toolbox. The networks consisted of three layers of fully interconnected neurons in the input layer, hidden layer and output layer. Neural networks with three different structures were considered. Network with structure $\# 1$ has five neurons in the input layer corresponding cutting parameters: cutting speed, feed rate and depth of cut, and cutting force signal, tangential force and feed force. Structure \#2 consisted of four neurons in the input layer corresponding cutting parameters: cutting speed, feed rate and depth of cut, and spindle motor power, while structure \#3 has six input variables: cutting speed, feed rate and depth of cut, spindle motor power and tangential force and feed force. In all the structures, the hidden layer consists of two layers, with the number of neurons in each layer varied 




Fig. 1: Experimental setup

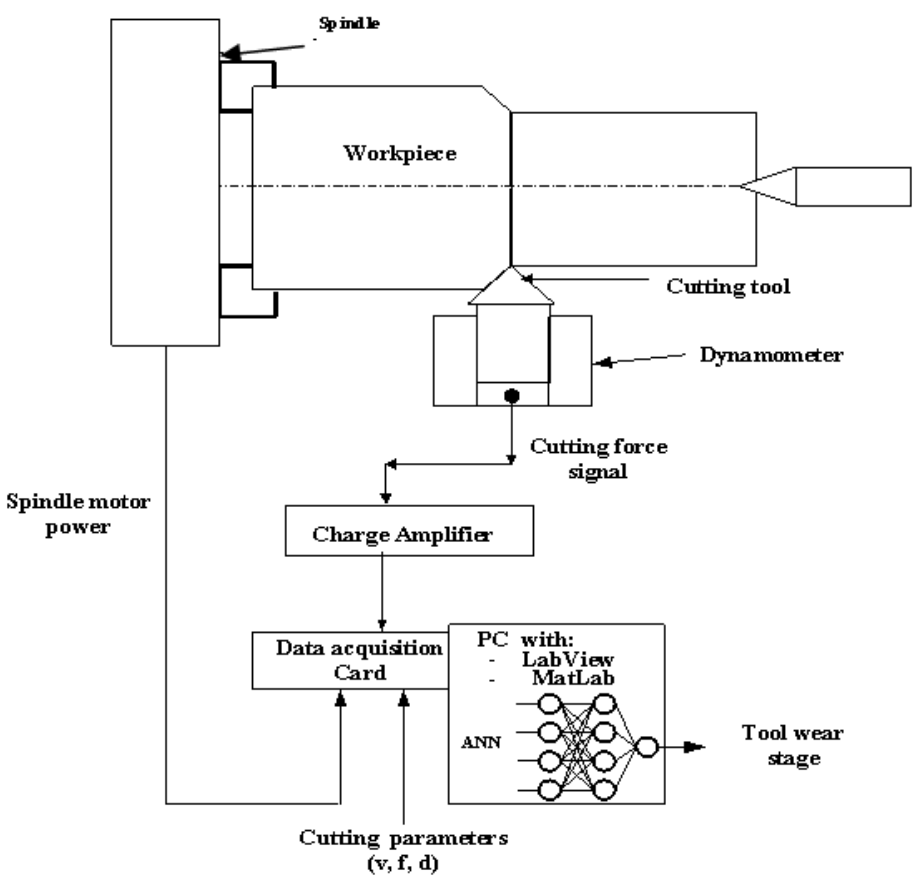

Fig. 2: Schematic of the experimental setup 
Table 4: Classifications of tool wear states

\begin{tabular}{lcc}
\hline \multirow{2}{*}{ Tool wear state } & \multicolumn{2}{c}{ VB $_{\max }$ range (mm) } \\
\cline { 2 - 3 } & Roughing & Finishing \\
\hline Light & $0.00-0.35$ & $0.00-0.20$ \\
Medium & $0.35-0.70$ & $0.20-0.40$ \\
Heavy & $>0.70$ & $>0.40$ \\
\hline
\end{tabular}

from 5 to 15 in the step of 5 , while the output layer consists of a single layer of 1 neuron corresponding to the predicted tool wear. The topologies of the networks are shown in Figure 3.

\section{Training of the ANN model}

Tangential sigmoid and linear transfer functions were used in the hidden and output layer. The resilient back-propagation training algorithm was chosen to train the network because of its fast convergence time in function approximation (Demuth and Beale, 2000). Randomised initial weights and biases were used for each neuron in all the layers and maximum number of iterations for training of the network was set at 30,000, learning rate was set at 0.55 , while Mean Square Error (MSE) of 0.005 was used as minimum network performance. Prior to the training process, the input/output dataset were normalised to values between \pm 1.0 . The training input/output dataset consisted of randomly selected 25 machining data from the roughing operations and 15 machining data from the finishing operations.

\section{Testing of the ANN model}

The accuracies of networks were tested with 9 (nine) machining data randomly selected from both rough and finish turning operations. The testing data sets for the roughing operation were chosen at cutting speeds of 90, 100 and $120 \mathrm{~m} / \mathrm{min}$ for each of the three wear stages, while data sets for finishing operation were chosen at cutting speeds of 100, 110 and 120 $\mathrm{m} / \mathrm{min}$. The network accuracy was determined by comparing the predicted and actual measured tool wear and the network percentage error was calculated as:
$P E=\frac{1}{N} \sum_{N=1}^{N} \frac{\left(a_{j}-t_{j}\right)}{t_{j}} \times 100$

Where $a_{j}$ is the network predicted tool wear at node $\mathrm{j}, \mathrm{t}_{\mathrm{j}}$ is the actual measured tool wear at node $\mathrm{j}, \mathrm{PE}$ is the network percentage error and $\mathrm{N}$ is the number of trials. Based on the network percentage error, the network performance was classified as follows:

- $\mathrm{PE}<0.0 \%$ Over-prediction (ANN predicted values above actual tool wear values)

- $\mathrm{PE}=0.0 \%$ Correct prediction (ANN predicted values equal to actual tool wear values)

- $\mathrm{PE}>0.0 \%$ Under-prediction (ANN predicted values below actual tool wear values)

\section{RESULTS AND DISCUSSION}

Electron scanned micrographs of typical wear land observed during the finish turning operations for light, medium and heavy wear stages are shown in Figure 4. It can be observed that flank wear is the predominant wear mode in high-speed turning of Ti-6Al-4V alloy. The cutting force signals acquired during the finish turning operation with cutting speed $(100 \mathrm{~m} /$ $\mathrm{min})$, feed rate $(0.15 \mathrm{~mm} / \mathrm{rev})$ and depth of cut $(0.5 \mathrm{~mm})$ for a medium worn cutting tool are shown in Figure 5. For this cutting condition, the tangential force $\left(\mathrm{F}_{\mathrm{z}}\right)$ was about two fold higher than the feed force $\left(\mathrm{F}_{\mathrm{x}}\right)$.

The actual and the ANN predicted tool wear using the network with structure \#3 (with the cutting speeds, feed rate, depth of cut, cutting forces and spindle motor power as input dataset) for finishing operations are presented in Table 5. The predicted values represent average 




a) Structure \#1

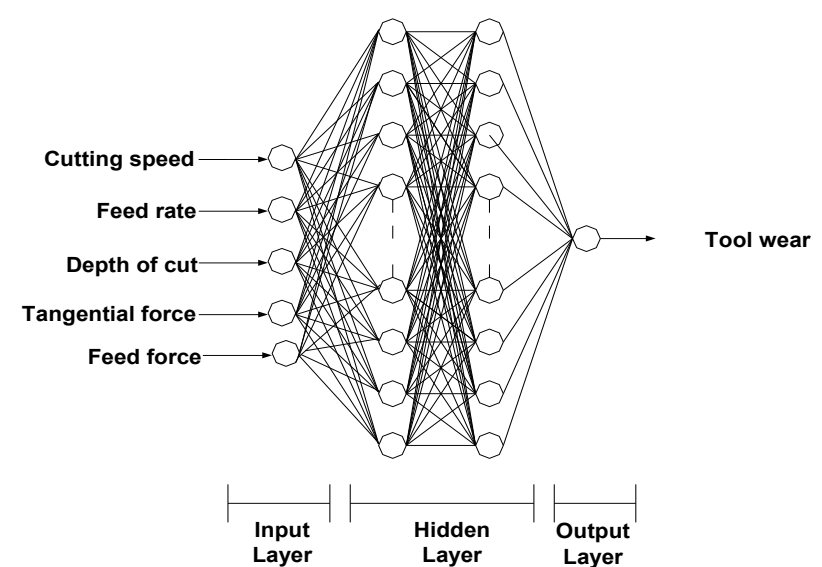

b) Structure \#2

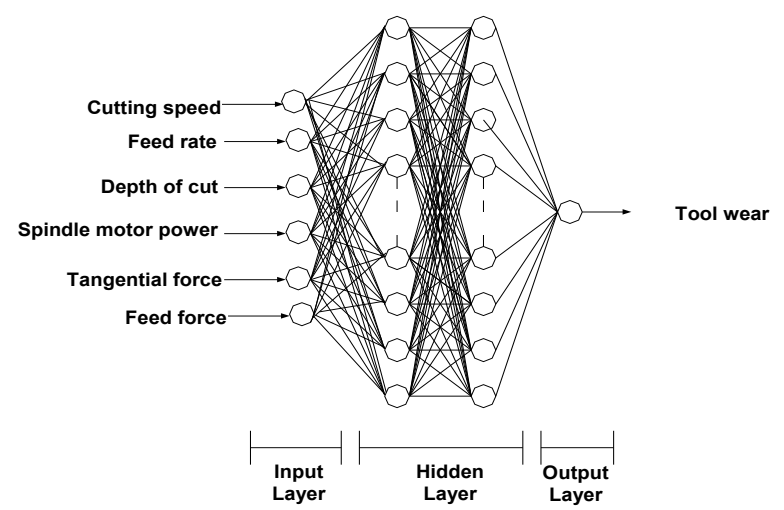

c) Structure \#3

Fig. 3: Topologies of the ANN for prediction of tool wear 


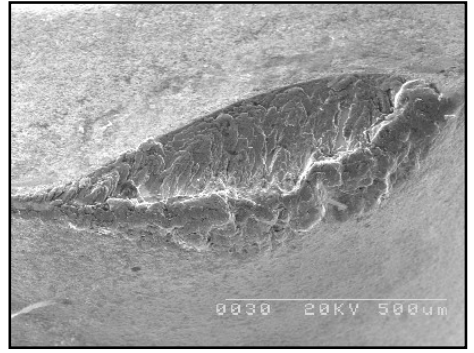

(a)



(b)

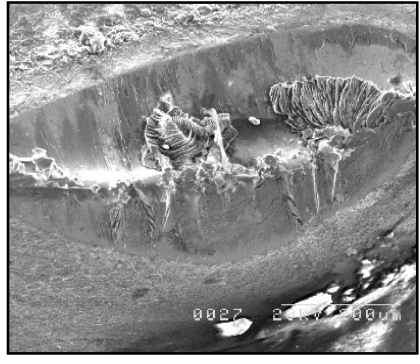

(c)

Fig. 4: Worn carbide inserts used in finish turning of Ti-6Al-4V alloy showing: (a) light wear; (b) medium wear; (c) heavy wear



Fig. 5: Cutting force signals acquired during the finish turning of Ti-6Al-4V alloy (cutting speed $=100 \mathrm{~m} / \mathrm{min}$, feed rate $=0.15 \mathrm{~mm} / \mathrm{rev}$ and depth of cut $=0.5 \mathrm{~mm}$

of 30 different trials where different random initial weights and biases were used in each trial. For finish turning operations (Table 5) percentage error of the model ranged between 14.08 and $12.62 \%$. The range of error obtained is within the general acceptable values for machining operations (Bernhard, 2002).

The variations in the neural network percentage error for rough turning of Ti-6Al-4V alloy using uncoated K10 carbide inserts are shown in Figure 6. Figure 6(a) shows variation in the network percentage error when the network was trained with only the machining speed and force signals. The variations when the network was trained with only machining speed and power consumption is represented in Figure 6 (b), while Figure 6(c) shows the variation when the network was trained with both machining speed, force and power consumption signals. It can be observed from Figures 6(a-c) that there were variation in the network performance at different levels of tool wear. The network percentage errors are consistently lower when the wear level is heavy, followed by light wear and finally medium wear. The network's ability to predict tool wear more accurately for heavy and light wear levels can be explained in part by the wear pattern of the cutting tool. The wear pattern of cutting tool is divided into three regions: initial breakdown; uniform wear rate; and rapid breakdown. The initial breakdown and rapid breakdown regions are known to exhibit much higher wear rate (Bernhard, 2002). Therefore, the machining parameters employed in this 
Table 5. Actual and ANN predicted tool wear for finishing operations.

\begin{tabular}{ccccc}
\hline \multirow{2}{*}{ Wear stage } & $\begin{array}{c}\text { Cutting speed } \\
(\mathbf{m} / \mathbf{m i n})\end{array}$ & Actual & ANN Predicted & Percentage error $(\%)$ \\
\cline { 3 - 5 } & 100 & 0.1040 & 0.1054 & 1.35 \\
Light & 110 & 0.1790 & 0.1538 & -14.08 \\
& 120 & 0.1220 & 0.1374 & 12.62 \\
\multirow{2}{*}{ Medium } & 100 & 0.2620 & 0.2850 & 8.78 \\
& 110 & 0.2590 & 0.2831 & 9.31 \\
& 120 & 0.3160 & 0.3006 & -4.87 \\
Heavy & 100 & 0.4230 & 0.4558 & 7.75 \\
& 110 & 0.4110 & 0.4616 & 12.31 \\
& 120 & 0.5510 & 0.5131 & -6.88 \\
\hline
\end{tabular}

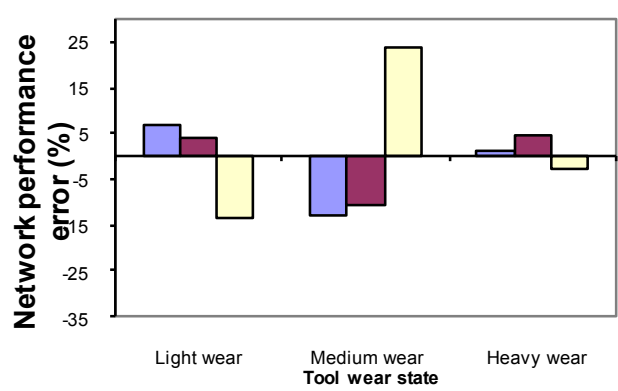

(a)

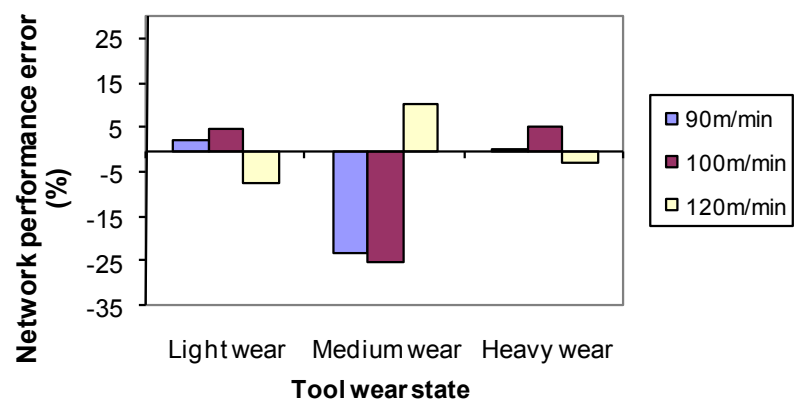

(c)

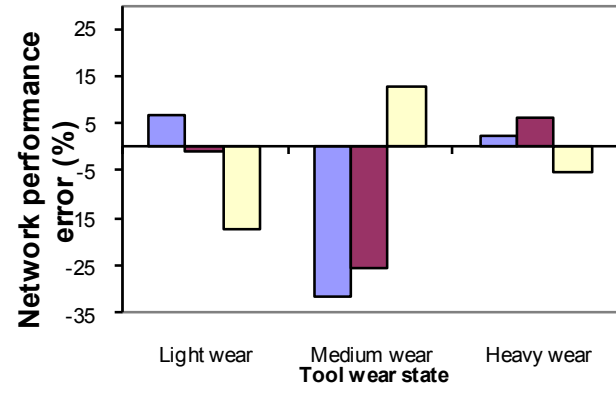

(b)

Fig. 6: Rough turning of Ti-6Al-4V: (a) cutting speed and force signals only; (b) cutting speed and spindle power only; (c) cutting speed, force and spindle power 
study tend to be more correlated to tool wear at the regions of rapid initial wear and accelerate final wear in comparison to the steady intermediate wear region. The network's ability to predict tool wear for heavy wear level corresponding to the rapid breakdown region appears to be systematically high due to accelerated wear rate in this region, followed by the prediction for light wear level corresponding to initial breakdown region with equally high rate of tool wear.

Figure 6(a) shows that the network predicts tool wear relatively well with percentage error ranging between 23.4 and $-13.8 \%$ for rough turning when trained with only the speed and force signals. From Figure 6(b), it can be seen that the performance of the network degraded with percentage error ranging between 12.8 and $31.4 \%$ when the network is trained with only speed and power consumption signals, while Figure 6(c) shows a general improvement in the network performance with percentage error ranging between 10.0 and $-25.2 \%$ when the network is trained with both speed, force and power consumption signals.

\section{CONCLUSIONS}

From analysis of results obtained in high-speed machining trials of Ti-6Al-4V alloy with uncoated carbide inserts at the cutting conditions investigated, the following conclusions can be drawn:

1. The predictive performance of a multilayer perceptron improves significantly for heavily worn tools than light and medium worn tools.

2. The use of cutting force signal as tool wear indicator has proved to be more efficient than power consumption signal.

3. Better tool wear prediction can be obtained by combining the cutting parameters, cutting force and power consumption signals.

\section{ACKNOWLEDGEMENT}

Dr. D. A. Fadare is grateful to the John D. and Catherine T. MacArthur Foundation Grant for the financial support given to the University of Ibadan for Staff Development Program under which this research work was carried out at the Machining Research Centre (MRC), London South Bank University, London, UK.

\section{REFERENCES}

Akangah, P. and Wang, K. (2006). "Intelligent condition monitoring of rotating machinery" Journal of Science and Technology (Ghana) 26(3):149-158.

Azimian, A.R. (2005). "Application of artificial neural network in pipe flow calculations" Proceedings of the 4th International Conference on Heat Transfer, Fluid Mechanics, and Thermodynamics, HEFAT 2005, Cairo, Egypt. (On CD) Paper AA1.

Bernhard, S. (2002). "On-line and indirect tool wear monitoring in turning with artificial neural networks: a review of more than a decade of research" Mechanical System. Signal Processing 16(4): 487-546.

Deiab, I., Assaleh, K. and Hammad, F. (2009). "On modelling of tool wear using sensor fusion and polynomial classifiers" $\mathrm{Me}$ chanical Systems and Signal Processing, 23(5): 1719-1729.

Demuth, H. and Beale, M. (2000). "Neural Network Toolbox User's Guild", Version 4 (Release 12). The MathWorks, Inc.

Dimla, D.E., Lister, P.M. and Leighton, N.J. (1997). "Neural network solutions to the tool condition monitoring problem in metal cutting- a critical review of methods", International Journal of Machine Tools and Manufacture 37(9): 1219-1242.

Ehmann, K.F., Kapoor, S.G., Devor, R.E. and Lazoglu, I. (1997). "Machining process modelling: a review" Journal of Manufacturing. Science \& Engineering 119: 655663.

Ezugwu, E.O., Arthur, S.J. and Hines, E.L. (1995). "Tool-wear prediction using artificial neural networks". Journal of Material Processing Technology 49: 255-264. 
Ezugwu, E.O., Fadare, D.A., Da Silva, R.B., Bonney, J. and Nelson, A.S. (2004). "Wear prediction of uncoated carbide tool during high speed turning of Ti-6V-4V alloy using artificial neural network". Advances in Manufacturing Technology, Proceedings of the Second International Conference on Manufacturing Research ICMR2004, Sheffield Hallam University, UK, 36-41.

Kuljanic, E., Totis, G. and Sortino, M. (2009). "Development of an intelligent multisensor chatter detection system in milling" $\mathrm{Me}$ chanical Systems and Signal Processing 23 (5): 1704-1718.

Lin, J.T., Bhattacharyya, D., Kecman, V. (2003). "Multiple regression and neural networks analyses in composites machining", Composites Science and Technology 63: $539-548$

Liu, T.L., Chen, W.Y. and Anantharaman, K.S. (1998). "Intelligent detection of drill wear". Mechanical System and Signal Processing 12(6): 863-873.

Picton, P. (2000) "Neural networks" $2^{\text {nd }}$ Edition. UK: Antony Rowe Ltd.

Portillo, E., Marcos, M., Cabanes, I. and Zubizarreta, A. (2009). "Recurrent ANN for monitoring degraded behaviours in a range of workpiece thicknesses" Engineering Applications of Artificial Intelligence, (In Press).

Rashed, F.S. and Mahmoud, T.S. (2209). "Prediction of wear behaviour of A356/ $\mathrm{SiC}_{\mathrm{p}}$ MMCs using neural networks" Tribology International 42(5): 642-648.
Sick, B. (2002). "On-Line and Indirect Tool Wear Monitoring in Turning with Artificial Neural Networks: A Review of more than a Decade of Research" Mechanical System and Signal Processing 16(4): 487-546.

Silva, R.G., Baker, K.J., Wilcox, S.J. and Reuben, R.L. (2000). "The adaptability of a tool wear monitoring system under changing cutting conditions" Mechanical Systems and Signal Processing 14: 287-298.

Silva, R.G., Reuben, R.L., Baker, K.J. and Wilcox S.J. (1998). "Tool wear monitoring of turning operations by neural network and expert system classification of a feature set generated from multiple sensors", $M e$ chanical Systems and Signal Processing 12: 319-332.

Wang, L., Mehrabi, G.M. and Kannatey-Asibu Jr, E. (2004). "Tool wear monitoring in reconfigurable machining systems through wavelet analysis". Engineering Research Center for Reconfigurable Machining Systems, University of Michigan. Web based publication, http://erc.engin.umich.edu/ publications/PubFiles/ TA3 /ProjR2/ LitaoNAMRC01.pdf - pp 1-7. (Accessed on June 2004)

Wilkinson, P., Reuben R.L., Jones, J.D.C, Barton, J.S., Hand, D.P., Carolan, T.A. and Kidd, S.R. (1999). "Tool wear prediction from acoustic emission and surface characteristics via artificial neural network" $\mathrm{Me}$ chanical Systems and Signal Processing 13 (6): 955-966. 